\title{
The Relationship Between Physician Assistant School Admissions Exam (PA-CAT) and Undergraduate Performance Measured by Science GPA and Cumulative GPA"
}

\author{
Scott Massey \\ Central Michigan University physician Assistant program, masse2sl@cmich.edu \\ Johnna Yealy \\ The University of Tampa, Department of Physician Assistant Medicine, jyealy@ut.edu \\ Rajat Chadha \\ University of Illinois at Urbana-Champaign, chadhar@illinois.edu \\ David Beck \\ University of Pittsburgh, Physician Assistant Studies Program, dbeck@pitt.edu
}

Follow this and additional works at: https://nsuworks.nova.edu/ijahsp

Part of the Educational Assessment, Evaluation, and Research Commons, and the Medicine and Health Sciences Commons

This Manuscript has supplementary content. View the full record on NSUWorks here: https://nsuworks.nova.edu/ijahsp/vol18/iss2/9

\section{Recommended Citation \\ Massey S, Yealy J, Chadha R, Beck D. The Relationship Between Physician Assistant School Admissions Exam (PA-CAT) and Undergraduate Performance Measured by Science GPA and Cumulative GPA". The Internet Journal of Allied Health Sciences and Practice. 2020 Jan 01;18(2), Article 9.}

This Manuscript is brought to you for free and open access by the College of Health Care Sciences at NSUWorks. It has been accepted for inclusion in Internet Journal of Allied Health Sciences and Practice by an authorized editor of NSUWorks. For more information, please contact nsuworks@nova.edu. 


\title{
The Relationship Between Physician Assistant School Admissions Exam (PA- CAT) and Undergraduate Performance Measured by Science GPA and Cumulative GPA"
}

\begin{abstract}
ABSTRACT

Purpose: Physician Assistant (PA) programs often set minimum GPA and graduate record examination (GRE) requirements for admission, citing that candidates with higher admission scores will perform better in the PA program. However, to date, there are limited published studies with inconsistent results that have investigated the validity of using these preadmission characteristics to predict performance in PA programs or on the Physician Assistant National Certifying Exam (PANCE). The development of a physician assistant college admission test (PA-CAT) that has predictive validity to determine PANCE success would give PA admissions committees an additional resource to make decisions. This study was conducted to determine the strength of the relationship between PA-CAT and undergraduate cumulative and science GPA. Methods: The PA-CAT is comprised of 180 questions covering 12 subject areas based on research identifying the relative importance of that subject to success in the PA curriculum. The exam was administered through a secured computer-based testing to 479 newly admitted PA students across the United States. Regression analysis was conducted with Rasch scale scores as the dependent variable and two independent variables (undergraduate GPA and undergraduate science GPA). Results: The PA-CAT Rasch scale scores are positively correlated with undergraduate GPA $(r=0.16)$ and undergraduate science GPA ( $r=0.22)$. Although these correlation coefficients are statistically significant (pConclusion:Early results from this research study demonstrates there is a statistically significant relationship between the PACAT and undergraduate science GPA in newly admitted PA students. Limitations of the study include the fact that students voluntarily took this exam without consequence. Further study is needed to determine if the exam can be generalized to the entire PA applicant pool thereby providing a valid instrument for admissions decisions.
\end{abstract}

\section{Author Bio(s)}

Scott Massey PhD PA-C is the director of the Central Michigan University physician Assistant program. Dr. Massey has 27 years of PA educational experience. He has published numerous research articles and has presented dozens of national and international presentations. He is also been involved with several international projects promoting the training of health workers

Rajat Chadha, PhD is a Measurement Specialist at the University of Illinois, Urbana-Champaign. He has extensive experience working as a psychometrician and statistician on international projects for organizations such as Howard Hughes Medical Institute, UNICEF, OECD, International Baccalaureate, and the Governments of Australia, Bangladesh and India.

David C. Beck MPAS, PA-C, DFAAPA is the Director of the University of Pittsburgh Physician Assistant Studies Program. He has been involved in several research projects throughout his career, and he is particularly interested in improving social justice, equity, and data-driven decision making into PA admissions processes.

Johnna Yealy PhD PA-C is the chair of the UTampa PA program. She has twice served as the president of the Tennessee Academy of PAs. She has presented at the AAPA, AAMC and PAEA national conferences and authored numerous peer reviewed journal articles as well as a textbook chapter. 


\title{
1IVAHSP \\ The Internet Joumnal of Allied Health Sciences and Practice \\ Dedicated to allied health professional practice and education
}

Vol. 18 No. 2 ISSN 1540-580X

\section{The Relationship Between PA School Admissions Exam and Undergraduate Performance Measured by Science GPA and Cumulative GPA}

\author{
Scott Massey ${ }^{1}$ \\ Joanna Yealy ${ }^{2}$ \\ Rajat Chadha ${ }^{3}$ \\ David Beck ${ }^{4}$
}

1. Central Michigan University

2. University of Tampa

3. University of Illinois at Urbana-Champaign

4. University of Pittsburgh

United States

\begin{abstract}
Purpose: Physician assistant (PA) programs often set minimum GPA and graduate record examination (GRE) requirements for admission, citing that candidates with higher admission scores will perform better in the PA program. To date, few published studies have investigated the validity of using these preadmission characteristics to predict performance in PA programs or on the Physician Assistant National Certifying Exam (PANCE), and these few studies have inconsistent results.. The development of the Physician Assistant College Admission Test (PA-CAT) that has predictive validity to determine PANCE success would give PA admissions committees an additional resource to make decisions. This study was conducted to determine the strength of the relationship between PA-CAT score and undergraduate science GPA and cumulative GPA. Methods: The PA-CAT is composed of 180 questions covering 12 subject areas based on research identifying the relative importance of that subject to success in the PA curriculum. The exam was administered through secured computer-based testing to 479 newly admitted PA students across the United States. Regression analysis was conducted with Rasch scale scores as the dependent variable and two independent variables (undergraduate GPA and undergraduate science GPA). Results: The PA-CAT Rasch scale scores are positively correlated with undergraduate GPA $(r=0.16)$ and undergraduate science GPA $(r=0.22)$. Although these correlation coefficients are statistically significant $(P<.001)$, the strength of the correlation is not strong. The regression coefficient of undergraduate science GPA is statistically significant $(P<.01)$. An examinee with an undergraduate science GPA 1 whole point higher (ie, 2.5 to 3.5) is expected to score 17 scale score points higher on the PA-CAT. This translates to about nine-tenths of the standard deviation of the sample scale scores. The regression coefficient for the undergraduate GPA is not statistically significant. Conclusion: Early results from this research study demonstrate there is a statistically significant relationship between the PA-CAT and undergraduate science GPA in newly admitted PA students. Limitations of the study include the fact that students voluntarily took this exam without consequence. Further study is needed to determine if the exam can be generalized to the entire PA applicant pool to provide a valid instrument for admissions decisions.
\end{abstract}

Keywords: physician assistant, admissions, examination, predict 
BACKGROUND AND SIGNIFICANCE/PRELIMINARY STUDIES

Physician assistants (PAs) are nationally certified and state-licensed medical providers who examine, diagnose, and treat patients of all ages with the supervision of a licensed physician. PAs are licensed in all 50 United States, the District of Columbia, and the majority of US territories. ${ }^{1}$ To be licensed to practice medicine as a PA, the individual must graduate from an academic program accredited by the Accreditation Review Commission on Education for the Physician Assistant (ARC-PA) and pass the Physician Assistant National Certifying Examination (PANCE) governed by the National Commission on Certification of Physician Assistants (NCCPA). Therefore, the overall goal of PA academic programs is to prepare students with the knowledge and skills necessary to complete these two milestones.

In response to the increased demand for PAs, there has been an increase in the number of PA educational programs. The vast majority (95\%) of these programs use the Centralized Application Service for Physician Assistants (CASPA) to process student applications. ${ }^{2}$ A recent report analyzed 10 years of student applicant data from the CASPA system and revealed that there was a $53 \%$ increase in the number of applicants per seat from 1.81 in 2002 to 2.77 in $2011 .{ }^{3}$ Researchers found relatively constant trends in the major demographic and academic features for applicants, but the average grade point average (GPA) of matriculated students increased from 3.39 to 3.49 , indicating an increasingly competitive applicant pool. These facts underscore this greater challenge PA educators have in choosing students from a larger, more academically qualified applicant pool.

Student achievement, indicated by completion of the academic program and passing the PANCE, is as important to the applicant as it is to the program. For students, success is paramount to reaching entry-level competency, gaining licensure, and beginning a medical career. Before investing time and money in an educational program, discerning applicants will examine the student success outcomes for various programs and consider these characteristics when choosing which program to attend. From an accreditation perspective, the ARC-PA requires programs to publish annual data regarding student achievement including program attrition and PANCE pass rate. Programs that report below $85 \%$ first-time PANCE pass rates must provide additional analysis of their PANCE performance to the ARC-PA. ${ }^{4}$ Therefore, PA faculty must assess applicants' potential to succeed and their subsequent performance, beginning with the selection of students at admission and continuing through each phase of the program, graduation, and taking the PANCE. As faculty select students for admission and monitor student progression, knowing what factors predict success in the program and on the PANCE is imperative in helping identify students at risk of failure. ${ }^{5}$

PA programs often set minimum undergraduate GPA and Graduate Record Examination (GRE) requirements for admission, citing that candidates with higher admission GPA and GRE scores will perform better in the PA program. Currently, the national average first-year PA class GPAs are as follows: overall undergraduate GPA (GPA-U): 3.56; undergraduate science GPA (GPA-Sc): 3.51 ; biology/chemistry/physics GPA: 3.46; and undergraduate non-science GPA: $3.61 .{ }^{6}$ However, to date, there are limited published studies that have investigated the validity of using these preadmission characteristics to predict performance in PA programs or on the PANCE. The few available studies demonstrate inconsistent results. The development of a physician assistant admission college entrance exam that has predictive validity to determine PANCE success would give PA admissions committees an additional resource to make admission decisions.

Review of PA admissions literature as it relates to the predictive ability of preadmission criteria reveals only a handful of results. The outcome variable of PANCE score was the variable of interest in all the studies, well established as one of the most important criteria to judge the success of a PA program. Students must successfully graduate from their accredited program to take this certifying examination, so graduation is inherent in this measure of success. Each of the studies used a retrospective design that included correlation and multiple regression to study the relationship between specific variables of preadmission characteristics and PANCE scores. Most of the studies reviewed a single program and generally included data from 2 to 3 cohorts of students, limiting the generalization of the results.

In an article authored by Higgins et al, admission variables as predictors of PANCE scores across six universities were explored. This study was the broadest in terms of the number and location of study subjects. The study included students who entered between 2003 and 2006 and graduated by 2008. The institutions included in the study were a mixture of both private and public, housed in liberal arts institutions and academic health centers. They found that 4 predicators made a significant contribution to the final regression equation: GPA, GRE Verbal, GRE Quantitative, and PACKRAT scores, with PACKRAT scores being the most consistent and best predictor of performance on the PANCE. ${ }^{7}$ The PACKRAT (Physician Assistant Clinical Knowledge Rating and Assessment Tool) is a standardized examination administered by the Physician Assistant Education Association to evaluate a student's medical knowledge before clinical rotations, shown to be predictive of performance on the PANCE. ${ }^{8}$ However, the PACKRAT is not a preadmission criterion, as it is only administered after the student has matriculated and completed at least half of their PA training program.

(C) The Internet Journal of Allied Health Sciences and Practice, 2020 
A 2013 study by Brown reviewed a single program over 3 graduating classes. Released in 2013, the study found that there was no correlation between PANCE performance and undergraduate GPA, science prerequisite GPA, or health care experience. PANCE scores were correlated with overall program GPA, PA pharmacology, and PA anatomy grade. ${ }^{9}$ This study relied solely on correlation analysis and was unique in that undergraduate GPA was not correlated to PANCE score. The study found that specific performance indicators while in PA education was more highly correlated with PANCE success.

The Andreeff study looked at PA students at the D'Youville College from 2006 through 2010. She found that higher first-attempt PANCE scores were predicted by higher undergraduate GPA and pathophysiology and biochemistry undergraduate coursework. ${ }^{5}$ She included preadmission criteria in the study, so the effect of performance while in PA training could not be assessed. Gender and age were not found to be predictive of PANCE score.

In 2001, Ennulat published a study that included data collected over 3 years at the Chatham University PA program. They found that all assessment measure variables they tested had significant positive correlations with performance on the PANCE. The variables that were included were a mixture of preadmission criteria (undergraduate GPA and GPA in prerequisite courses) and performance criteria while in the program, including the PACKRAT, a summative examination, and the average of all multiple choice examinations given while in the didactic phase of the program. All the predicting variables were positively correlated with PANCE score, but the most highly predictive were the performance variables while in PA education. The study authors developed a predictive equation that would allow program faculty to identify at-risk students. ${ }^{8}$

The most recently published research is by Butina et al (2017) out of the University of Kentucky. They reviewed data from 9 cohorts of a single institution $(\mathrm{N}=469)$. They completed a pathway analysis to identify early those students at risk of failing the PANCE in their PA program. Their path analysis indicated that grades from foundational coursework were the strongest early predictors of PANCE performance, accounting for over half of the variance in PANCE performance. Other direct influencers were the undergraduate GPA and the GRE Verbal score. ${ }^{10}$

A dissertation study completed by Yealy in 2017 reviewed 11 cohorts of data $(N=528)$ from the University of Oklahoma Health Science PA program. The purpose of this study was to find 1 or more significant relationships among the preadmission characteristics and performance variables of program GPA, passing the PANCE, and score on the PANCE. Yealy found that the total GRE score, the last 60 [undergraduate credits] GPA, and the science GPA were predictive of the total PA program GPA. The last 60 GPA and PA program GPA were most predictive of passing or failing the PANCE. The PA program GPA was predictive of the PANCE score. ${ }^{11}$

Studies have shown that scores on standardized cognitive tests are good predictors of academic performance in graduate programs. ${ }^{12}$ Generally speaking, the predictive validity of standardized exams is highly similar across most disciplines. However, the predictive value of the GRE for graduate health professions success is less well understood, and the study of this issue has revealed inconsistent results. ${ }^{13}$ As of 2017, the GRE was an admissions criterion in 59.9\% of PA programs. ${ }^{6}$ Both the GRE Verbal and GRE Quantitative were found to be predictive of the PANCE score in one published study and for the GRE-Verbal in the Butina study; no such predictive value was found in research completed for a master's thesis. ${ }^{7,10,14}$ The data are not overwhelming and are inconsistent regarding the relationship between the GRE and PANCE scores. Furthermore, Hocking found that the majority of programs were not using GRE scores as recommended by the Educational Testing Services (ETS). ${ }^{15}$ Because current data on the predictive value of the GRE are incongruent, Hocking recommended that programs reassess using the GRE as an admission prerequisite. This issue is further complicated by the fact that underrepresented student groups are known to have lower standardized test scores on the GRE. ${ }^{16}$ As can be seen from the literature review, using a standardized exam as an admission criterion is common but controversial. Studies are inconsistent in their ability to predict academic success in graduate health professions programs based on the standardized exam alone.

Other health professions have developed standardized pre-admission exams to assist admissions committees in the arduous process of selecting applicants most likely to be academically successful in their training and ultimately pass their board exams. One such exam is the Pharmacy College Admission Test (PCAT), launched in 1974, which consists of 5 multiple-choice subtests (verbal ability, biology, chemistry, reading comprehension, and quantitative ability) and a writing subtest. Research undertaken in 2011 sought to determine the predictive validity of the PCAT and other variables for student success in the first year of pharmacy training. ${ }^{17}$ PCAT scores and entering GPAs for 22 pharmacy programs were reviewed, and it was found that PCAT scores and entering GPAs both showed moderate predictive validity in indicating candidates likely to succeed in the first year of pharmacy school. This finding is consistent with studies conducted since the introduction of the PCAT that have found that the test is a moderate to strong predictor of subsequent performance, with predictive validity statistics comparable to those of other standardized admission tests commonly used by graduate and professional schools. ${ }^{12,13}$

( The Internet Journal of Allied Health Sciences and Practice, 2020 
A meta-analysis of published studies to determine the predictive validity of the Medical College Admission Test (MCAT) on medical school performance and medical board licensing exams was conducted in 2007. The study found that the predictive validity of the MCAT ranges from small to medium for both medical school performance and medical board licensing exam measures. The medical profession is challenged to develop screening and selection criteria with improved validity that can supplement the MCAT as an important criterion for admission to medical school. ${ }^{18}$

\section{STUDY AIMS}

The aim of this study is to determine if the PA-CAT is predictive of success in PA training as measured by passing the PANCE. The study will be a joint effort between the external coinvestigators and one of the larger testing companies in the United States, Exam Master. This 2-year study will focus on the following research questions:

1. To determine the correlational strength between the Physician Assistant College Admission Test (PA-CAT) scores and undergraduate science GPA and cumulative undergraduate GPA in newly enrolled PA students.

2. To determine the predictive validity of the Physician Assistant College Admission Test (PA-CAT) scores and other cognitive variables for student success in the first year of graduate physician assistant programs.

3. To determine the predictive validity of the Physician Assistant College Admission Test (PA-CAT) scores and other cognitive variables for student success in the final GPA of graduate physician assistant programs.

4. To determine the predictive validity of the Physician Assistant College Admission Test (PA-CAT) scores and other cognitive variables for student success on the Physician Assistant National Certifying Examination (PANCE).

5. To determine if there are statistically significant differences in scores among student ethnicities on the Physician Assistant College Admission Test (PA-CAT) scores.

For this publication, the authors will present the data related to research question number 1 and 5 :

1. Does the PA-CAT have a statistically significant correlation with the subject's undergraduate GPA and undergraduate science GPA?

5. Are there statistically significant differences in scores among student ethnicities on the PA-CAT?

\section{Administrative Organization}

The principal investigators of this study are the program directors of the physician assistant programs at the University of Tampa and Central Michigan University. They have entered into an agreement with the PA-CAT developers (Exam Master) to analyze the data and produce predictability tables. This article presents an analysis of the initial data gathered during the first phase of this longitudinal study. Exam Master is a corporation with over 400 health science education clients, serving the physician assistant educational community since 2004. Exam Master's study, testing, and review resources are in use by approximately 100 PA programs in the United States. Additionally, Exam Master provides a secure platform for exam delivery that has met the University of Tampa's (participating pilot program) IT security team's qualifications to allow for student testing. The study was reviewed by the University of Tampa's IRB committee and determined to be exempt.

\section{METHODS}

\section{Instrumentation}

The PA-CAT is an assessment instrument developed by Exam Master. There are 180 questions in the basic medical science component, comprising the following distribution of questions: anatomy (15\%), physiology $(15 \%)$, general chemistry $(10 \%)$, general biology $(10 \%)$, microbiology $(10 \%)$, genetics $(10 \%)$, biochemistry $(5 \%)$, organic chemistry $(5 \%)$, psychology $(5 \%)$, sociology $(5 \%)$, statistics $(5 \%)$, and medical terminology $(5 \%)$.

Distribution of questions was determined after a subcommittee of experts from both the basic science and the PA education professions conducted a meta-analysis of the most common distribution of prerequisite courses. The questions were constructed by content experts with experience in test item construction.

Rasch measurement is a rigorous and unified approach to addressing multiple aspects of data, including investigating the quality of individual questions, investigating the reliability and validity of measures, and enabling conversion of raw scores (total number of questions answered correctly) into interval-level measures (distances between units on the scale remain same across the measurement scale). These interval-level measures are more appropriate for many statistical analysis approaches than raw scores. ${ }^{19}$ Raw scores are ordinal-level data where the score intervals are unequal. For example, on a 180-question exam, the difference between a score of 175 and 180 and the difference between a score of 85 and 90 do not reflect the same difference in person ability. On the other hand, the same difference in interval-level measures reflects the same difference in person ability on

(C) The Internet Journal of Allied Health Sciences and Practice, 2020 
any part of the measurement scale. Moreover, when comparing raw scores, only inferences about greater or lesser can be drawn without the magnitude of the difference between 2 scores; when comparing interval-level measures, decisions about the magnitude of difference between measures can be made.

The Rasch Model is a probabilistic model that estimates person ability and item difficulty measures on the same measurement scale and assumes that the probability of a person answering an item correctly is determined only by the difference between the person ability and item difficulty measures. This prescriptive model defines the expected responses if measurement is to be achieved. As part of quality control, outfit statistics are calculated to assess if the question-level and person-level responses match the model expectations. Person-level response data fit the Rasch Model well, with $99.4 \%$ of the person outfit statistics within the desirable range of 0.5 to 1.5 . Item response data also fit the Rasch Model well, with $98.5 \%$ of the question outfit mean-square fit statistics within the desirable range of 0.8 to $1.2 .{ }^{19}$ If the data fit the Rasch Model well, as is the case here, items contribute meaningfully to the measurement of person ability, and the person measures are on the interval scale; they are more appropriate for statistical analysis than the raw scores.

Individual student results from the PA-CAT were not disseminated to the 22 participating programs; the program faculty were blinded to individual student results throughout this longitudinal study to intentionally prevent any intervention or remediation to be administered to lower-scoring individuals and to maintain the integrity of the study.

Reliability of person measures was good at 0.83 , which implies that, of the total observed variance, $83 \%$ is the true variance, indicating that there is high probability that the persons estimated to have higher measures actually have higher measures than the persons estimated to have lower measures.

\section{Study Design}

This study's design is retrospective. The study participants were students entering PA programs in the 2018-2019 year. The sample size was 479. The first outcome point is at the end of the matriculation cycle for the participating programs (generally August), when all initial PA-CAT exams have been completed. Demographic and incoming admission characteristics (GPAs) will be analyzed to determine any correlation with the PA-CAT.

\section{Subject Selection}

The study sample is a convenience sample consisting of all the incoming matriculating students of the study site PA programs. The potential research subjects were consented according to IRB requirements. Participation rates range from approximately $5 \%$ to $100 \%$ per participating program.

\section{Recruitment Procedures}

The study participants were asked to participate in taking the PA-CAT. All students signed informed consent following procedures from the University of Tampa IRB. The students completed the exam using the Exam Master platform and an account number that was provided after consent. The individual program test site administrators provided Exam Master with demographic and incoming GPA for each participant. The study population in this publication was derived from 22 PA programs, representative of all geographic locations in the United States, including Midwest, Northeast, Middle Atlantic, Southeast, Mountain West, and West Coast. The IRB clearly communicated to programs that the results of the PA-CAT would not be distributed to programs, and individual students did not receive any feedback about performance. These communications were necessary to avoid any change in behavior toward lower-scoring students. PA students were required to sign the IRB consent form, which clearly stated that the results of this examination would not be made available to the student or to the faculty member.

\section{RESULTS \\ PA-CAT Test Scores}

A total of 479 examinees were administered versions 1.0,1.1, and 1.2 of the PA-CAT exam. All versions were intended to measure the same construct and included the same 12 subjects. Questions in versions 1.1 and 1.2 were improved based on the analysis of data from the preceding version. Version 1 was administered to 86 examinees, version 1.1. to 233 examinees, and version 1.2 to 160 examinees. Person measures (scale scores) were estimated using the Rasch Model in Winsteps 4.2.0. ${ }^{1}$ As discussed earlier, critical distinctions between the Rasch scale scores and raw scores (number of questions correct) are that the scale scores are interval-level measures where distances between units on the scale remain same across the measurement scale and the scale scores are appropriate for statistical analysis. Even though the 3 sets of examinees were administered different versions of the exam, the Rasch Model estimates person measures on the same measurement scale just as different thermometers can measure the temperature on the same scale.

(C) The Internet Journal of Allied Health Sciences and Practice, 2020 
Scale scores were estimated so that scores without decimals do not leave behind any information that may be useful in identifying individual difference among examinees. ${ }^{2}$ The lowest performing examinee answered 37 questions correctly, and the highest performing examinee answered 128 questions correctly. Both mean and median scale score were 490 , and the standard deviation was 20 , with a minimum of 414 and maximum of 548 . Mean and median scale score of 490 for examinees is close to the mean question difficulty measure of 500 , which indicates that the difficulty of the questions matches the examinees well, and the exam is neither too difficult nor too easy. The scale scores were approximately normally distributed (Figure 1).

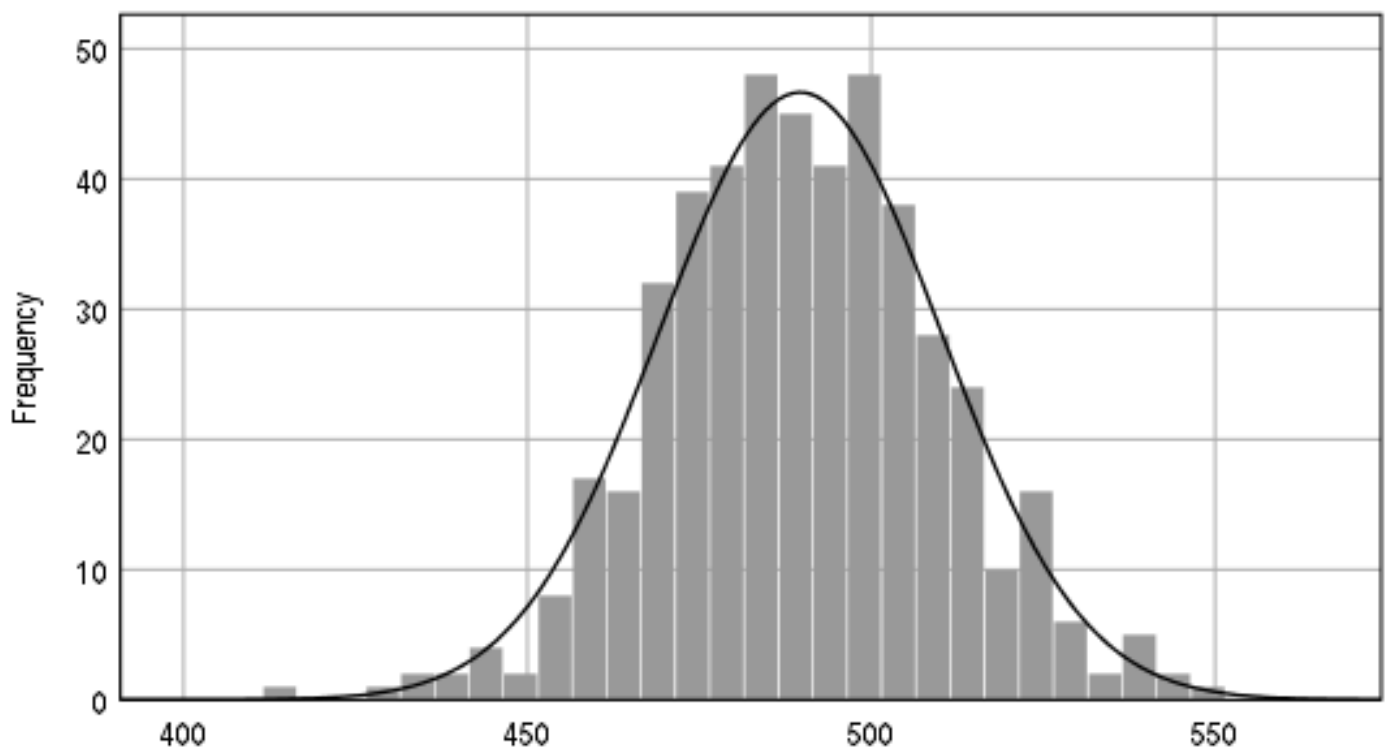

Scale Score

Figure 1. Distribution of Scale Scores

\section{Examinees}

Approximately $74 \%$ of the examinees identified as female. Mean age of examinees was 26 years, and median age was 24 years. The minimum age of examinees in the sample was 20 years, and the maximum age was 55 years. Race/ethnicity of examinees is reported in Table 1 below.

Table 1: Ethnicity of Examinees

\begin{tabular}{|l|c|}
\hline \multicolumn{1}{|c|}{ Race/ethnicity } & $\%$ \\
\hline White / Caucasian Non-Hispanic & $57.8 \%$ \\
\hline Hispanic / South or Central American & $21.8 \%$ \\
\hline Asian / Pacific Islander & $11.1 \%$ \\
\hline Black / African American & $4.0 \%$ \\
\hline Multiracial & $1.6 \%$ \\
\hline Native Alaskan & $0.2 \%$ \\
\hline No Report or Unknown & $3.6 \%$ \\
\hline
\end{tabular}


Relationship Between Undergraduate GPA, Undergraduate Science GPA, and PA-CAT Scale Scores

Correlation

The Pearson correlation coefficient between undergraduate GPA $(M=3.57, S D=0.23)$ and PA-CAT scale scores was $0.16(P$ $<.0001$ ) (Figure 2). The Pearson correlation coefficient between undergraduate science GPA ( $M=3.54, S D=0.27$ ) and PA-CAT scale scores was $0.22(P<.001)$ (Figure 3$)$.

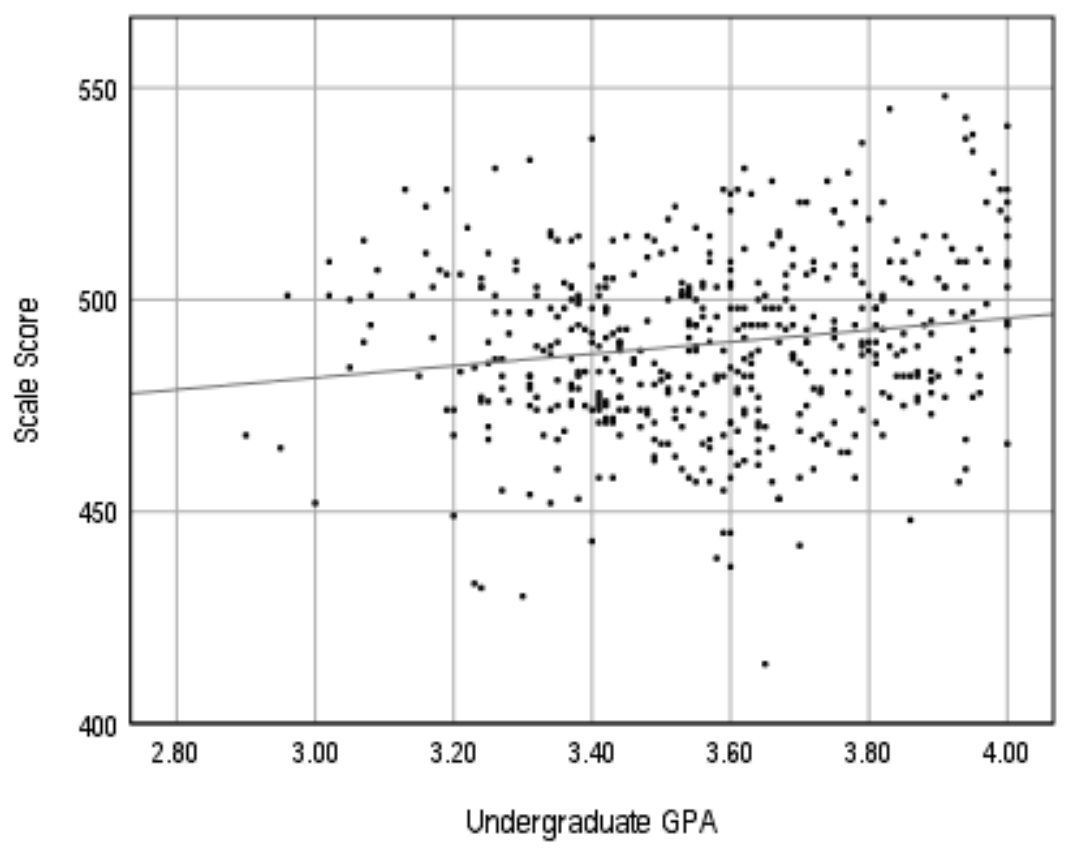

Figure 2. Scale Score and Undergraduate GPA

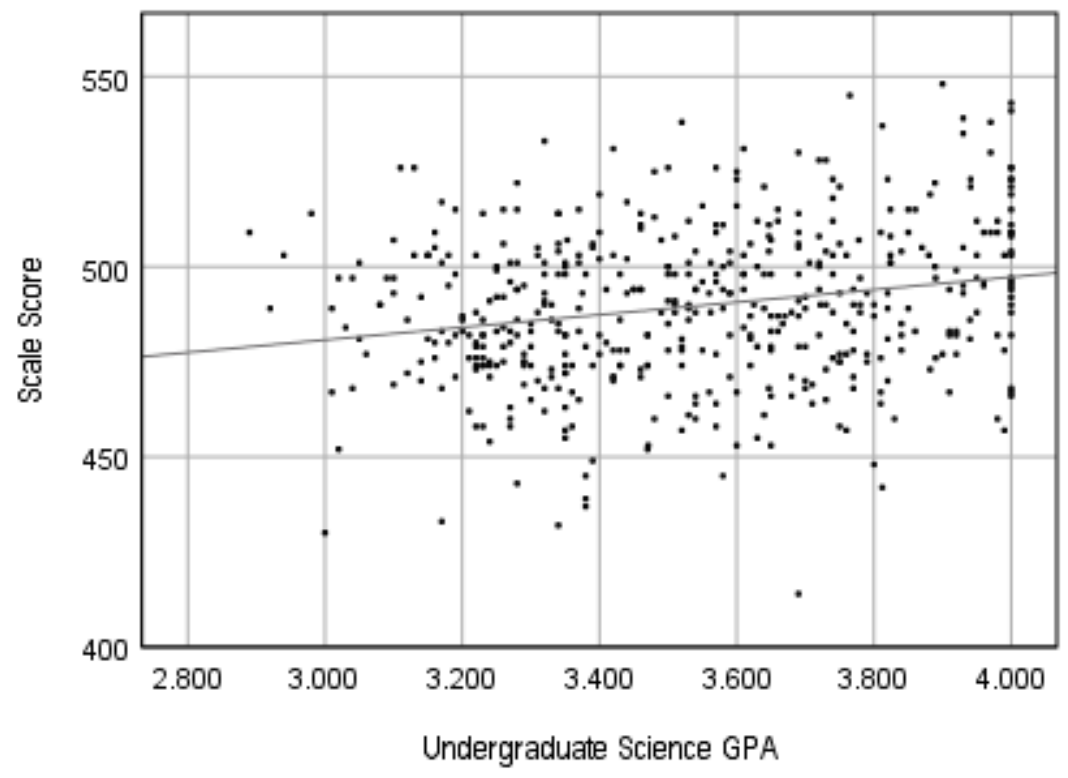

Figure 3. Scale Score and Undergraduate Science GPA 


\section{Multiple Regression}

Multiple linear regression analysis was conducted using IBM SPSS Statistics 25 with PA-CAT scale scores as the predicted variable and undergraduate GPA and undergraduate science GPA as two predictor variables. ${ }^{3}$ All assumptions underlying the multiple regression analysis were met, implying that findings of the analysis are reliable. The regression model was statistically significant $\left(R^{2}=0.044, F(2476)=12.04, P<.001\right)$ with a multiple correlation of 0.22 . Multiple correlation coefficient ranges from 0 to 1 with higher values indicating better predictability of the predicted variable based on the predictor variables. In addition to statistical significance tests, which inform the probability of observing a relationship by chance, a measure of practical significance that emphasizes the strength of the relationship is effect size. The two predictor variables explain $4.4 \%$ of the variance in scale scores with a small effect size (Cohen's $\mathrm{f}^{2}=0.05$ ). ${ }^{4}$ In other words, the strength of the relationship between the undergraduate GPA and undergraduate science GPA is small. It was found that undergraduate science GPA significantly predicted PA-CAT scale scores $(\beta=17.39, P<.01)$. An examinee with an undergraduate science GPA of 1 whole point higher is expected to score 17 scale score points higher on the PA-CAT exam, about nine-tenths of the standard deviation of the current sample's scale scores. Undergraduate GPA did not significantly predict PA-CAT scale scores $(\beta=-1.32, P=0.827)$ after accounting for undergraduate science GPA.

\section{Difference Between Scale Scores by Ethnicity}

An analysis of variance was conducted to investigate whether the mean scale scores of examinees with different ethnicity are statistically significantly different. As noted in Table 1, the test population was predominantly the following groups: White / Caucasian Non-Hispanic, 57.8\%; Hispanic / South or Central American, 21.8\%; and Asian / Pacific Islander 11.1\%., Only these 3 groups were considered for this analysis because the number of examinees are very small for the other groups. There are no statistically significant differences between the mean scale scores for the 3 groups $(F(2405)=2.04, P=0.131)$. Levene's test of homogeneity of variance is not statistically significant $(P=0.117)$, indicating that the equal variance assumption for the 3 groups is tenable and that no adjustment is needed.

\section{DISCUSSION}

Strengthening the admissions process and selecting the candidates most likely to successfully complete a program are important goals of PA educators. The statistically significant predictive relationship between undergraduate GPA and PA-CAT scale scores supports the ongoing development of the PA-CAT and investigation of its other relationships, especially to indicators of student performance in PA programs. Similarly, the equal variance assumption among the 3 largest ethnic groups of examinees is promising, as equity and social justice are and will continue to be of importance to the research team and the PA-CAT developers. This study was based on the original exam blueprint; however, the next version of the exam will include an additional section that measures student empathy. The inclusion of the Jefferson Scale of Empathy (JSE) as a component of the PA-CAT was the result of feedback from numerous conversations with directors of PA programs, who indicated that, in addition to academic preparedness, they were looking to assess noncognitive attributes as part of the admissions process. Many felt that empathy was an important characteristic for PA students and practitioners to be successful clinicians. Empathy was viewed as a component of maturity and of professionalism.

Members of the PA-CAT Advisory and Research Committees also concluded that broadening the scope of the PA-CAT to measure important nonacademic applicant characteristics such as empathy would help provide a more holistic admissions exam that would be less disadvantageous to underrepresented population groups. Many PA programs include in their mission an aspect related to increasing diversity and the number of underrepresented minority PA students.

\section{Limitations}

Limitations in this study include the sampling methods and several external variables. The convenience sampling method resulted in a population composed only of students who had been selected and matriculated into PA programs. Further, the sizes of the ethnic groups of the study population were the result of convenience; representation was not ensured. Regarding the external variables, participants voluntarily sat for the PA-CAT without the grading consequences or the targeted preparation experienced by applicants taking admissions tests like the GRE or MCAT.

\section{Implications for Further Research}

Further investigation is needed to determine the correlational strength and predictive validity of the PA-CAT in terms of PA program performance.. Because of the limitations of the study, generalizing to the entire PA applicant pool will not be possible until the exam is tested with the intended population. The limitations of the study were primarily because the test was given to a pre-selected group of matriculated students deemed superior to the bulk of applicants to these PA programs due to a combination of academic and nonacademic attributes.

(C) The Internet Journal of Allied Health Sciences and Practice, 2020 
The researchers have chosen to administer the test to existing PA students who are newly matriculated into a PA program. There would be ethical considerations to administer this test to the general population before further internal validation. The researchers had concerns that this information could influence admissions decisions prematurely; this methodology was chosen as the best available means to research this test instrument

Another limitation is that the GRE was not chosen as a comparison variable. Not all the programs require the GRE, so this was not used as another variable in this study.

\section{CONCLUSION}

Completion of this 2-year longitudinal study is necessary to determine if the PA-CAT represents a viable instrument for admissions purposes. The findings of this initial exploratory study were encouraging, especially regarding the statistical significance of the regression coefficient of undergraduate science GPA $(P<.01)$, which validates that an examinee with an undergraduate science GPA 1 whole point higher is expected to score 17.39 scale score points higher on the PA-CAT. This comparison translates to about nine-tenths of the standard deviation of the sample scale scores. Further study is needed by testing the actual admissions population before the determination can be made that the PA-CAT can be used as a valid admissions tool.

\section{References}

1. AAPA. What is a PA? American Academy of Physician Assistants. 2019. https://www.aapa.org/what-is-a-pal. Accessed March 27, 2019.

2. PAEA. CASPA - The Central Application Service for Physician Assistants. Physician Assistant Education Association. 2019. https://paeaonline.org/caspal. Accessed March 27, 2019.

3. McDaniel MH. Central Application Service for Physician Assistants Ten Year Data Report 2002-2011. Journal of Physician Assistant Education. 2016:17-23.

4. ARC-PA. Change Forms/Required Reports. The Accreditation Review Commission on Education for the Physician Assistant. http://www.arc-pa.org/accreditation/resources/program-change-forms/. Accessed March 27, 2019.

5. Andreeff R. Predictors of student success on the physician assistant national certifying examination. The Journal of Physician Assistant Education. 2014:36-39.

6. PAEA. Program Report 33. Washington, DC: Physician Assistant Education Association; 2018. PAEA 33.

7. Higgins R, Moser S, Dereczyk A, et al. Admission variables as predictors of PANCE scores in physician assistant programs: A comparison study across universities. Journal of Physician Assistant Education. 2010;21(1):10-17.

8. Ennulat $\mathrm{CW}$, Garruba C, Delong DF. Evaluation of multiple variables predicting the likelihood of passage and failure of PANCE. The Journal of Physician Assistant Education Association. 2011;22(1):7-18.

9. Brown GI. Correlations Between PANCE Performance, Physician Assistant Program Grade Point Average, and Selection Criteria. The Journal of Physician Assistant Education. 2013;24(1):42-44.

10. Butina M, Wyant AR, Cardom R. Early Predictors of Students at Risk of Poor PANCE Performance. Journal of Physician Assistant Education. 2017:45-48.

11. Yealy J. Roadmap for Success: The Application of Predictive Analytics to the Physician Assistant Education Admissions Process. [dissertation]. for Cypress, CA: Trident University International; 2017.

12. Kuncel NR, Hezlett SA. Standardized tests predict graduate students' success. Science. 2007:1080-1081. doi:10.1126/science.1136618

13. Kuncel NR, Credé M, Thomas LL, et al. A meta-analysis of the validity of the pharmacy college admission test (PCAT) and grade predictors of pharmacy student performance. American Journal of Pharmaceutical Education. 2005:69(3).

14. Miranda CM. The Relationship of Selected Admission and Program Variables and the Success of Marietta College Physician Assistant Student Performance on the Physician Assistant National Certifying Examination. (master's thesis). Marietta, Ohio: Marietta College; 2006.

15. Hocking JA, Piepenbrock K. Predictive ability of the Graduate Record Examination and its usage across physician assistant programs. The Journal of Physician Assistant Education. 2010;21(4):18-22.

16. Bleske-Rechek A, Kingsley B. Trends in GRE scores and graduate enrollments by gender. Intelligence 46 . 2014:25-34.

17. Meagher DG, Pan T, Perez CD. Predicting performance in the first year of pharmacy school. American Journal of Pharmaceutical Education. 2011:75(5). 
18. Donnon T, Paolucci EO, Violate $C$. The predictive validity of the MCAT for medical school performance and medical board licensing examinations: A meta-analysis of the published research. Academic Medicine. 2007:82(1):100-106.

19. Bond TG, Fox CM. Applying the Rasch Model: Fundamental Measurement in the Human Sciences. 2nd ed. New York, NY: Routledge; 2013. 